\title{
A magnetic guide to purify radical beams
}

Cite as: J. Chem. Phys. 149, 174201 (2018); https://doi.org/10.1063/1.5053656

Submitted: 24 August 2018 . Accepted: 17 October 2018 . Published Online: 02 November 2018

Jutta Toscano (D), Christopher J. Rennick (D), Timothy P. Softley (D), and Brianna R. Heazlewood
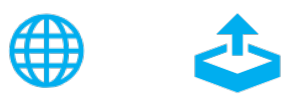

Export Citation

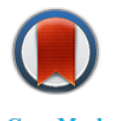

CrossMark

\section{ARTICLES YOU MAY BE INTERESTED IN}

Manipulating hydrogen atoms using permanent magnets: Characterisation of a velocityfiltering guide

Review of Scientific Instruments 90, 033201 (2019); https://doi.org/10.1063/1.5078573

Perspective: Computational chemistry software and its advancement as illustrated through three grand challenge cases for molecular science

The Journal of Chemical Physics 149, 180901 (2018); https://doi.org/10.1063/1.5052551

Slow photoelectron velocity-map imaging of cold $C_{7}^{-}$and $C_{9}{ }^{-}$

The Journal of Chemical Physics 149, 174306 (2018); https://doi.org/10.1063/1.5054792

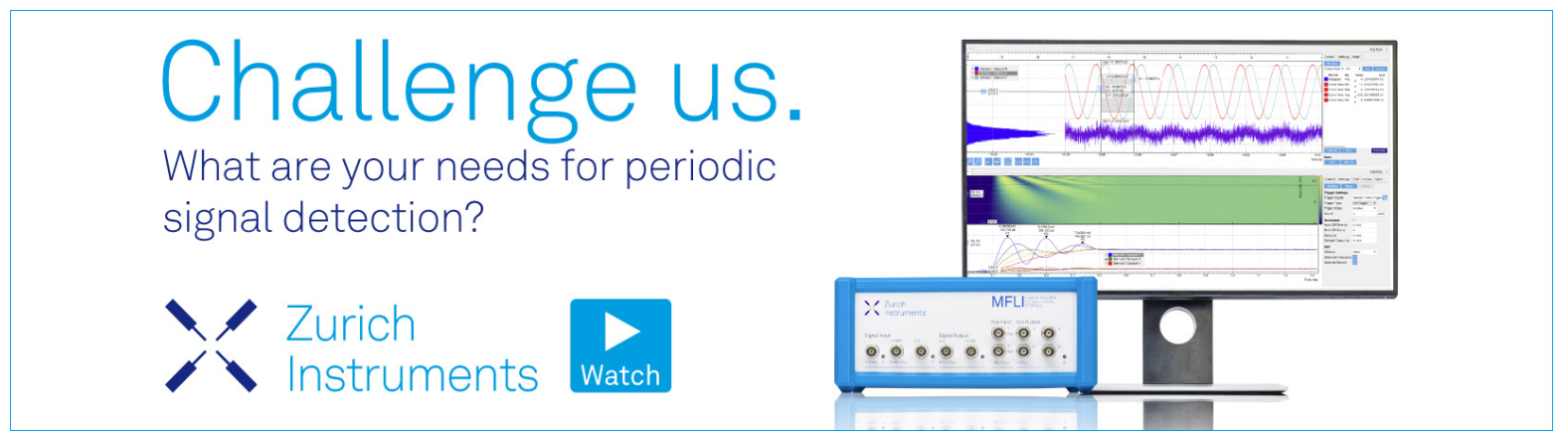




\title{
A magnetic guide to purify radical beams
}

\author{
Jutta Toscano, ${ }^{1}$ Christopher J. Rennick, ${ }^{2}$ Timothy P. Softley, ${ }^{3}$ and Brianna R. Heazlewood ${ }^{1}$ \\ ${ }^{1}$ Physical and Theoretical Chemistry Laboratory, Department of Chemistry, University of Oxford, \\ South Parks Road, Oxford OX1 3QZ, United Kingdom \\ ${ }^{2}$ National Physical Laboratory, Teddington, Middlesex TW11 OLW, United Kingdom \\ ${ }^{3}$ University of Birmingham, Edgbaston, Birmingham B15 2TT, United Kingdom
}

(Received 24 August 2018; accepted 17 October 2018; published online 2 November 2018)

\begin{abstract}
Generating a controllable and pure source of molecular free-radicals or open-shell atoms has been one of the primary barriers hindering the detailed study of radical processes in the laboratory. Here, we introduce a novel magnetic guide for the generation of a pure beam of velocity-selected radicals-a tuneable source that will enable the study of radical interactions with exceptional control over the properties of the radical species. Only radicals with a selected velocity are transmitted through the guide; all other components of the incoming beam (radical species traveling at other velocities, precursor molecules, and seed gas) are removed. The guide is composed of four Halbach arrays-hexapolar focusing elements - and two skimming blades. The relative positions of these components can be adjusted to tune the properties of the resulting beam and to optimise transmission for a given velocity. Experimental measurements of Zeeman-decelerated $\mathrm{H}$ atoms transmitted through the guide, combined with extensive simulations, show that the magnetic guide removes $99 \%$ of $\mathrm{H}$-atoms traveling outside the narrow target velocity range. Published by AIP Publishing. https://doi.org/10.1063/1.5053656
\end{abstract}

\section{INTRODUCTION}

Highly reactive species, such as ions and radicals, are abundant in many complex gas-phase environments-from very cold (the upper atmosphere, the interstellar medium) to very hot (plasmas, combustion systems, discharges) settings. The careful study of reactive collisions involving these highly reactive species, over a wide temperature range, is thus highly important for the accurate modeling of gas phase systems. In order to precisely and accurately study the kinetics and dynamics of radical reactions in the laboratory, one must satisfy a daunting set of conditions. Such studies require (i) a pure source of the radical reactant and any other reaction partners, with control over the properties of these reactants, (ii) sufficient number densities of each reactant species such that an appreciable number of reactive collisions will occur on an appropriate experimental time scale, and (iii) highly sensitive detection techniques, to monitor the reactions. Such requirements are exceedingly challenging to meet. Advances in ion trapping and laser cooling techniques mean that a diverse range of ionic reactants can now be routinely prepared in the cold regime - for example, within Coulomb crystals - and the reactions of these ions can be sensitively detected. ${ }^{1-3}$ By contrast, generating a controllable and pure source of molecular free-radicals or open-shell atoms has been one of the primary barriers hindering the detailed study of radical processes in the laboratory. Radical reactants are formed in very low densities under typical laboratory conditions and can also be extremely difficult to purify.

A velocity-selected radical beam source, with a wide range of velocity tunability and quantum state selectivity, could be utilised in a diverse range of applications. For example, the interactions between surfaces and radicals are key to understanding vapour deposition rates and film growth on surfaces.
Hydrogen radicals are known to play a role in the plasma deposition of silicon-based materials; they can bond to surfaces and occupy binding sites, react with molecules already deposited onto the surface (hindering film growth), and dissociate molecules prior to surface deposition. ${ }^{4}$ A detailed understanding of these complex and competing processes requires precise studies with velocity-controlled hydrogen atom beams. In the case of ion-radical collisions, where both reactants are highly reactive species, there is a paucity of experimental data. While reaction rate constants and branching ratios have been measured for literally thousands of different reactions between ions and stable (non-radical) species, using techniques such as selected ion flow tube (SIFT), ion cyclotron resonance (ICR), and reaction kinetics in uniform supersonic flow (CRESU),${ }^{5-7}$ experimental studies on ion-radical reactions are extremely limited. Measurements of the branching ratios and temperature dependencies of ion-radical reactions are almost non-existent.

\section{MAGNETIC GUIDE REQUIREMENTS}

In a Zeeman decelerator, pulsed magnetic fields serve to decrease the forward velocity of a beam of paramagnetic particles. ${ }^{8,9}$ However, the beam emerging from a Zeeman decelerator is typically composed of a mixture of decelerated, partially decelerated, and undecelerated particles, in addition to precursor molecules and seed gas particles. If one wishes to use a Zeeman-decelerated beam to study radical reactions with controlled collision velocity and chemical composition, it must undergo further manipulation prior to entering the interaction region. In our work, a magnetic guide is employed to purify the post-deceleration beam such that only the decelerated radicals reach the interaction region for collisional studies. 
The requirements of such a guide are that it only transmits a beam of pure radicals with a narrow velocity distribution and that this velocity is tuneable to facilitate the study of, for example, ion-radical reactions as a function of collision energy. The combination of a Zeeman decelerator with a linear Paul ion trap offers a highly promising approach for the study of a diverse range of ion-radical reactions under cold, controlled conditions-provided that only the decelerated radical species of interest are transmitted into the ion trap region. The long trapping times and single-ion sensitivity that has been demonstrated with Coulomb-crystallized ensembles make them an ideal medium for the examination of such processes.

Pure radical beams have been produced by photodetachment of an electron from negative ions following mass and velocity selection. ${ }^{10}$ However, the applicability of this method is limited to species that readily accept a negative charge and does not allow for the purification of an existing radical beam. A different approach consists in coupling magnetic or electric deflection with mechanical barriers. ${ }^{11}$ Straight magnetic and electrostatic deflectors have successfully been employed to spatially separate the ortho from the para spin isomer of water ${ }^{12}$ and the cis from the trans conformer of 3 -aminophenol, ${ }^{13}$ respectively. A pure beam of state-selected NO radicals has been obtained by combining an electrostatic hexapole state-selector with a beamstop and a diaphragm. ${ }^{14}$ In such an approach, a $2 \mathrm{~mm}$ beamstop is placed in the middle of the hexapole-where the radicals of interest are most de-focused-and is centred on the beam axis, to block the carrier gas; a $2 \mathrm{~mm}$-diameter diaphragm is placed after the hexapole to remove any remaining carrier gas. Although very high state purity (99\%) is achieved, fine manipulation of the velocity distribution of the NO radicals is not feasible. Bent magnetic guides consisting of a series of permanent hexapole or octopole magnets have been shown to guide neutral beams of metastable neon ${ }^{15}$ and lithium ${ }^{16}$ atoms, respectively, and merged beam experiments have very successfully employed magnetic benders to direct paramagnetic particles onto the same axis as a beam of polar molecules. ${ }^{17,18}$ A design for a bent magnetic guide composed of hexapole magnets combined with current carrying wires has been proposed, ${ }^{19}$ where permanent hexapole magnets in a Halbach array configuration $^{20,21}$ [Fig. 1(a)] serve to deflect the decelerated particles away from the beam axis whilst current carrying wires enhance the velocity resolution and quantum-state selectivity of the
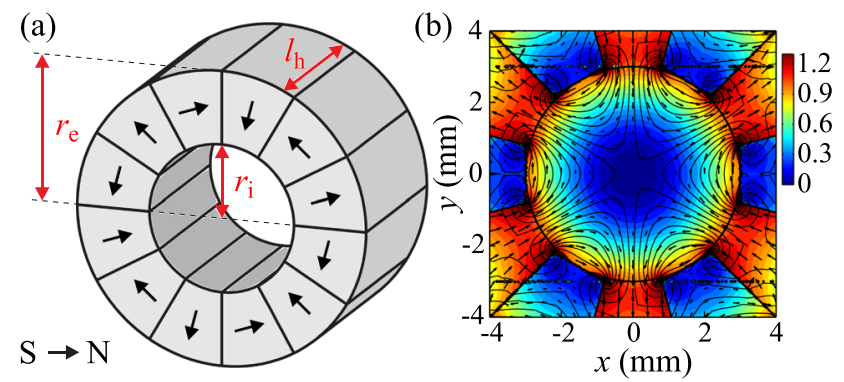

FIG. 1. (a) Schematic representation of one Halbach array of permanent magnets in hexapolar configuration. (b) Magnetic field magnitude (in Tesla) in the $x y$ plane for a Halbach array with remanence $B_{0}=1.3 \mathrm{~T}$, internal radius $r_{i}=3 \mathrm{~mm}$, external radius $r_{e}=6 \mathrm{~mm}$, and thickness $l_{h}=7 \mathrm{~mm}$. guided particles. The zero magnetic field at the centre of the arrays [Fig. 1(b)] is optimal for guiding particles in lowfield-seeking quantum states, as such particles minimise their Zeeman energy by traveling through the centre of the arrays. Hexapole magnets in a Halbach array configuration are particularly suitable here due to their characteristic strong magnetic fields within the cylinder and weak magnetic fields outside it, which minimises interference with other electromagnetic components of the experiment. Similar arrays have been employed to improve the transverse confinement of particles in between Zeeman-deceleration stages ${ }^{22}$ and are proposed in a design for a hydrogen storage ring. ${ }^{23}$

Here, we take inspiration from previous work ${ }^{19}$ and propose a novel magnetic guide: using Halbach arrays to deflect and collimate the decelerated particles off the main beam axis and around an on-axis blade that stops any faster or undeflected species, before returning the decelerated species back to the main beam axis again. Such a design increases both the practicality and flexibility of the guide. While originally developed to serve as a filter for Zeeman-decelerated radical beams, the magnetic guide reported in this work is immensely versatile. It could, for example, operate as a standalone radical beam filter-producing radical beams with a tuneable and narrow velocity distribution from a collimated effusive source-making the device relevant for a wide range of potential applications.

\section{METHODS}

The Zeeman decelerator apparatus has been described before (see Ref. 24) and is thus only briefly summarised here. Ground state hydrogen atoms are formed by photolysis of supersonically expanded ammonia seeded in krypton gas. The resulting mixture of precursor molecules, seed gas, hydrogen atoms, and other ammonia photofragments passes through a skimmer before flying through the 12-stage Zeeman decelerator, which selectively slows down $\mathrm{H}$ atoms in low-field-seeking quantum states. Post-deceleration, hydrogen atoms are resonantly ionised (via $2+1$ REMPI at $243 \mathrm{~nm}$ ) and detected with microchannel plates (MCPs). By scanning the delay between the photolysis and ionisation lasers, flight time traces can be collected. As described in Ref. 25, final velocities down to $75 \mathrm{~ms}^{-1}$ can be obtained with the current setup. Three-dimensional particle trajectory simulations are used to reproduce and interpret the experimental results (see Ref. 25).

The simulated beam of $\mathrm{H}$ atoms entering the Zeeman decelerator replicates the experimental distribution of positions and velocities; the resulting post-decelerator beam is subsequently flown through the magnetic guide. The dimensions of all components (skimmers, decelerator coils, Halbach arrays, blades) are explicitly specified in the simulations. Properties of the magnetic field within the Zeeman decelerator coils are derived from the analytical solution for a current loop, as described in Ref. 25. The Radia software package ${ }^{26-28}$ is employed to model the magnetic fields arising from the Halbach arrays. Specifying the dimensions and magnetisation of the array, the software returns a three-dimensional grid of magnetic-field values as a function of position inside 
and outside the array. During the propagation of the particles, the magnetic field present at any arbitrary position is calculated by linear interpolation between the nearest points within the grid. The fields from all elements are summed vectorially to obtain the total magnetic field at any given position. A velocity Verlet algorithm integrates the equations of motion every $100 \mathrm{~ns}$, and particles are propagated through the apparatus until they encounter a surface (such as a skimmer, decelerator coil, Halbach array, or blade) or reach the detection region. The detection region at the end of the magnetic guide is a circle with a $1.3 \mathrm{~mm}$ radius to match the acceptance of a linear Paul ion trap, for future ion-radical collision studies. To generate time-of-flight ( $\mathrm{ToF}$ ) profiles for comparison with the experimental traces, the detection laser is modelled as a Gaussian in the $z$ and $y$ dimensions, with the simulated signal given by the number of $\mathrm{H}$ atoms that pass through the laser detection volume within a selected time window.

Decelerated particles can be separated from other components of the beam using a single Halbach array shifted off axis. In order to focus and return these decelerated particles to the initial beam axis, several Halbach arrays can be arranged in series. The arrays have a velocity-dependent focal length that is controlled by magnetisation, internal radius, and thickness-similar to a wavelength-dependent optical focal length. Simulations evaluating the effect of remanence $B_{0}$, internal radius $r_{i}$, external radius $r_{e}$, and thickness $l_{h}$ of the arrays on focal length indicate that Halbach arrays with $B_{0}=1.3 \mathrm{~T}, r_{i}=4 \mathrm{~mm}, r_{e}=7 \mathrm{~mm}$, and $l_{h}=5 \mathrm{~mm}$ are suitable to target $\mathrm{H}$ atoms with final velocities between 125 and $200 \mathrm{~ms}^{-1}$ - the target range for our current Zeeman decelerator. Hence Halbach arrays with these properties are chosen for the guide.

Using trajectory simulations, we have determinated that the optimal magnetic guide, as depicted in Figs. 2(a) and
TABLE I. Simulated focal lengths corresponding to a series of target velocities in the operational range of the guide. These are determined by simulating the trajectories of ten particles flying through one Halbach array with longitudinal velocity equal to the target velocity and no transverse velocity. The particles are equally spaced in the transverse direction between $\pm 1 \mathrm{~mm}$ with respect to the beam axis.

\begin{tabular}{lllll}
\hline \hline Target velocity $\left(\mathrm{ms}^{-1}\right)$ & 125 & 150 & 175 & 200 \\
Focal length $f(\mathrm{~mm})$ & 5.0 & 7.2 & 9.6 & 12.4 \\
\hline \hline
\end{tabular}

2(b), is composed of four Halbach arrays and two skimming blades. The first array from the left deflects the slow particles up, the second collimates the deflected beam, the third brings it back to the beam axis, and the fourth array collimates the beam again. The distance between the midpoints of the first two and that of the second two arrays is twice the focal length, $2 f$ (see Table I). Within each pair, the two arrays have the same vertical shift, which is larger for the first pair $(s 12=+1.0 \mathrm{~mm})$ than for the second pair $(s 34=+0.6 \mathrm{~mm})$ [see Fig. 2(b)]. There are two blades, placed after the second and fourth arrays, that serve to physically block the undeflected components of the beam. The blades penetrate beyond the central axis of the decelerator (the end of the first blade is at $y=b 1=+1.4 \mathrm{~mm}$ and the second blade extends down to the beam axis $y=b 2=+0.0 \mathrm{~mm}$ ) such that they overlap in the $y$ dimension to leave no direct line-of-sight between the decelerator and the ion trap. Hence, the blades can fully skim off all the radical species that are not traveling at the target velocity, in addition to any seed gas and precursor molecules that reach the end of the decelerator. Only the radicals with a specific target velocity, corresponding to the specific focal length of the guide, follow the correct trajectory and pass through the guide unhindered.

Different target velocities can be addressed by altering the separation between the first and second arrays and between the

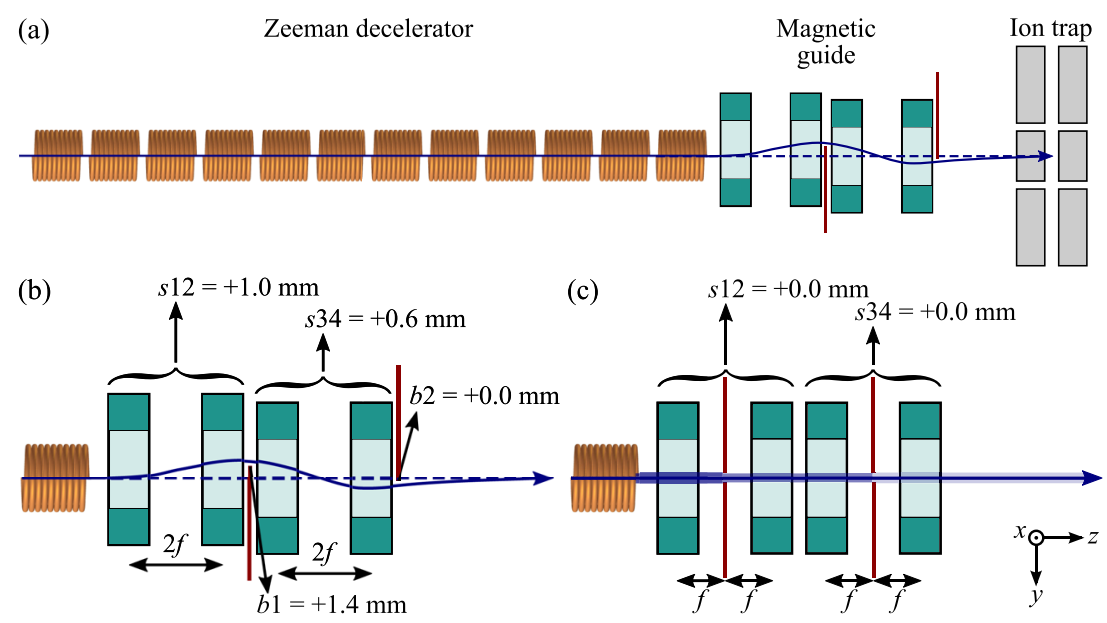

FIG. 2. (a) Schematic representation of the Zeeman decelerator combined with the magnetic guide and an ion trap. The solenoid coils are shown in orange, the Halbach arrays in green and the ion trap rods in grey (all in side view). The red vertical lines represent the retractable blades whilst the blue arrows represent the trajectories of the deflected (solid line) and undeflected (dashed line) particles. Geometry (b) of the shifted guide proposed here and (c) of its linear equivalent. The labels $s 12$ and $s 34$ correspond to the vertical shift of the first two Halbach arrays and of the second two arrays, respectively. In (b), $b 1$ is the $y$ position of the top end of the first blade and $b 2$ is the $y$ position of the bottom end of the second blade. The distance between the midpoint of arrays 1 and 2 and that of arrays 3 and 4 is equal to $2 f$, where $f$ is the focal length of the Halbach array for the velocity of interest. The blue arrows represent the trajectories of the deflected (solid line) and undeflected (dashed line) particles. In (c), the distance between the midpoint of each array and the nearest pinhole (in red) is equal to $f$. The blue arrow represents the trajectories of all particles whilst the superimposed shaded lines represent the gradual decrease in the number of faster-moving particles. 
third and fourth arrays, to account for the focal length variation with velocity. This is achieved by translating the second and fourth arrays along the $z$ axis. Additionally, the blades can be retracted or extended to tune the purity of the emerging beam: increasing the overlap in the $y$ dimension of the two blades improves the velocity selectivity of the guide but also decreases the number of transmitted particles. The compromise between the velocity selectivity and number of transmitted particles is also crucial in the choice of the vertical shifts of the Halbach arrays: a larger vertical shift of the first pair of arrays leads to a narrower velocity distribution of the transmitted beam, at the expense of a lower number of transmitted particles.

The various degrees of freedom in the magnetic guidethe positions of Halbach arrays two and four (with respect to arrays one and three), the $y$-axis shift of the two pairs of arrays, and the $y$-axis penetration of the retractable bladesare controlled by vacuum compatible, micrometer adjustable actuators. Four linear actuators independently shift each pair of arrays and each blade in the $y$ dimension whilst two rotary actuators turn two bevelled gears that are coupled to a threaded spindle to move the second and fourth arrays in the $z$ dimension. In this way, the parameters of the guide can be adjusted whilst the apparatus remains under vacuum.

\section{RESULTS}

\section{Trajectory simulations}

The velocity filtering of a beam of hydrogen atoms passing through the guide after deceleration to a final velocity of $200 \mathrm{~ms}^{-1}(f=12.4 \mathrm{~mm})$ is illustrated in Fig. 3 using trajectory simulations. Particles with the target velocity (top panel) are deflected up and then down by the arrays; they avoid both skimming blades and reach the target region (gray line at $z=350 \mathrm{~mm}$ ). By contrast, faster-moving $\mathrm{H}$ atoms are completely skimmed off by the blades (middle panel) and slowermoving $\mathrm{H}$ atoms are partially skimmed off and partially deflected away from the target region (bottom panel). Of the hydrogen atoms that reach the target region following deceleration when the guide is not present, only $1.9 \%$ of the beam is within the target velocity range $\left(v_{z}=200 \pm 10 \mathrm{~ms}^{-1}\right)$, with 98\% of the beam composed of faster particles and $0.1 \%$ of slower particles. After passing through the magnetic guide, simulations indicate that $99 \%$ of the hydrogen atoms traveling outside the target velocity range have been removed. Simulations also show a significant $(62 \%)$ increase in the number of target particles transmitted to the detection region, owing to the focusing properties of the Halbach arrays. The purification achieved results in a very narrow longitudinal velocity distribution of the guided $\mathrm{H}$-atom beam of $205 \pm 9 \mathrm{~ms}^{-1}$. The average transverse velocity of the particles reaching the target region after passing through the guide is simulated to be $7.1 \mathrm{~ms}^{-1}$, in contrast to an average transverse velocity of $2.6 \mathrm{~ms}^{-1}$ in the absence of the guide. It is geometrically not possible for the non-magnetic species (precursor and seed gases) to pass through the guide unhindered by the blades, and therefore, $100 \%$ of these species are removed from the beam. In practice, a differential-pumping stage is needed between the guide

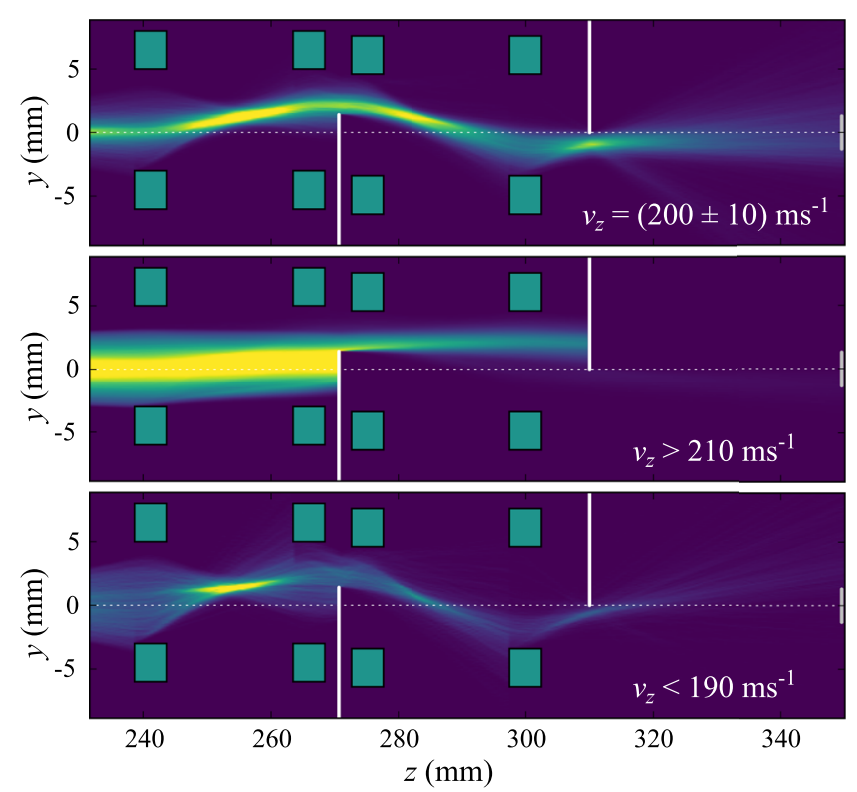

FIG. 3. Three-dimensional numerical particle trajectory simulations of a beam of $1 \mathrm{~s}{ }^{2} S_{1 / 2}$ hydrogen atoms flying through the guide. The incoming beam has already passed through a 12-stage Zeeman decelerator with a target final velocity of $200 \mathrm{~ms}^{-1}$ and can be divided into three contributions: particles traveling at the target velocity $\left( \pm 10 \mathrm{~ms}^{-1}\right)$, particles traveling faster than the target velocity, and particles traveling slower than the target velocity. The trajectories of the target particles $\left(v_{z}=200 \pm 10 \mathrm{~ms}^{-1}\right.$, top panel), faster particles $\left(v_{z}>210 \mathrm{~ms}^{-1}\right.$, middle panel $)$, and slower particles $\left(v_{z}<190 \mathrm{~ms}^{-1}\right.$, bottom panel) through the magnetic guide are shown, with intensity ranging from low (purple) to high (yellow). The green rectangles represent the Halbach arrays in cross section, the white solid lines represent the blades, and the gray line at $z=350 \mathrm{~mm}$ represents the detection region. The dotted horizontal white line shows the decelerator axis ( $z$ axis) on which the beam is initially centred. Only the hydrogen atoms traveling within the narrow target velocity range are directed into the detection region.

and the ion trap to prevent the skimmed particles from reaching the target region after bouncing off the blades.

A linear equivalent of the magnetic guide described above [see Fig. 2(c)] could be implemented, with the four Halbach arrays collinear and centred on the decelerator axis $(s 12=s 34$ $=+0.0 \mathrm{~mm}$ ). In place of blades, pinholes could be placed at the focal points of the target velocity (that is, between the first and second array and between the third and fourth array), to block out the unwanted components of the beam. Such a linear design, while perhaps more intuitive than the approach adopted in this work, is significantly less efficient at purifying the beam. Even with a very small pinhole diameter of $0.5 \mathrm{~mm}$, the percentage of $\mathrm{H}$ atoms with the target velocity after the guide is only $20.9 \%$, as the resulting beam is still mainly composed of faster-traveling $\mathrm{H}$ atoms $(75.4 \%)$ that remain along the beamline axis. Furthermore, these values do not include the contribution to the transmitted beam of any precursor or seed gases traveling along the centre of the apparatus. This is because the pinholes are (necessarily) centred on the beam axis; even with a very small diameter and when positioned at the focal point for the target velocity, pinholes will admit a large number of unwanted particles, given that the latter are several orders of magnitude more numerous than the target particles. The shifted guide proposed here outperforms its linear equivalent [Fig. 2(c)] due to the breaking of the $x y$ symmetry which, leaving no direct line of sight through the guide, is 
essential to skim off all of the unwanted particles and achieve a high purity of slow $\mathrm{H}$ atoms. A linear design only achieves a gradual purification of the beam and thus would require a large number of stages to achieve a comparable purification to that obtained by the shifted design employed in this work and would ultimately transmit far fewer target-velocity particles.

The shifted design also has several advantages over the bent design previously proposed. ${ }^{19}$ Bending the decelerated particles away from the beam axis would require one to change the radius of the bend every time a different velocity is desired or a different species is to be guided. One would also need to move the ion trap with the guide in order to study ion-radical collisions, which is impractical from an experimental point of view. The new design circumvents this practical difficulty by maintaining a collinear arrangement of the trap with respect to the decelerator, as well as allowing for the tuning of the target velocity in situ, without having to break the vacuum. Another alternative to using a magnetic guide to steer the radicals' trajectories would be to use a mechanical chopper or shutter. Using a chopper to select the part of the beam that is allowed to pass through based on arrival time would not give a sufficiently narrow velocity distribution and would also inevitably transmit some precursor and seed gas particles. A mechanical shutter could be employed to physically block out unwanted elements in the beam - and such an approach is taken by several research groups (including ours) in combination with, for example, a Stark decelerator. The limited lifetime of mechanical shutters operating under ultrahigh vacuum conditions and the finite opening time of the shutter vanes (approximately $750 \mu$ s for fast-opening designs) limit the timeframe over which experiments can be recorded and the range of velocities that can be addressed. As such, mechanical shutters are a less-than-ideal alternative.

\section{Experimental verification}

Experimental ToF traces-recorded with and without the magnetic guide in place after the decelerator-are shown in Fig. 4. In the absence of the guide, the ToF trace has three peaks, arising from $\mathrm{H}$ atoms that have not been decelerated, $\mathrm{H}$ atoms that have only been partially decelerated, and finally the fully decelerated $\mathrm{H}$ atoms (from shorter to longer timeof-flight, respectively). When the guide is placed after the decelerator, only the fully decelerated $\mathrm{H}$ atoms are transmitted, as predicted by the simulations (Fig. 4). Particles that contribute to the first two ToF peaks no longer reach the detection region. The experimental $\mathrm{ToF}$ traces are in excellent agreement with the simulated ToF profiles.

The versatility of the magnetic guide presented here allows for the target velocity of $\mathrm{H}$ atoms to be tuned from $200 \mathrm{~ms}^{-1}$ down to $125 \mathrm{~ms}^{-1}$ by simply adjusting the position of the second and fourth Halbach arrays (achieved using rotary actuators and thus without the need for breaking the vacuum) in combination with using a Zeeman decelerator pulse sequence optimised for the desired velocity. This is successfully demonstrated in the bottom panel of Fig. 4, where the experimental ToF traces clearly exhibit a single peak that moves to later arrival times as the velocity of the $\mathrm{H}$ atoms that are transmitted is sequentially lowered. The extent to which the blades

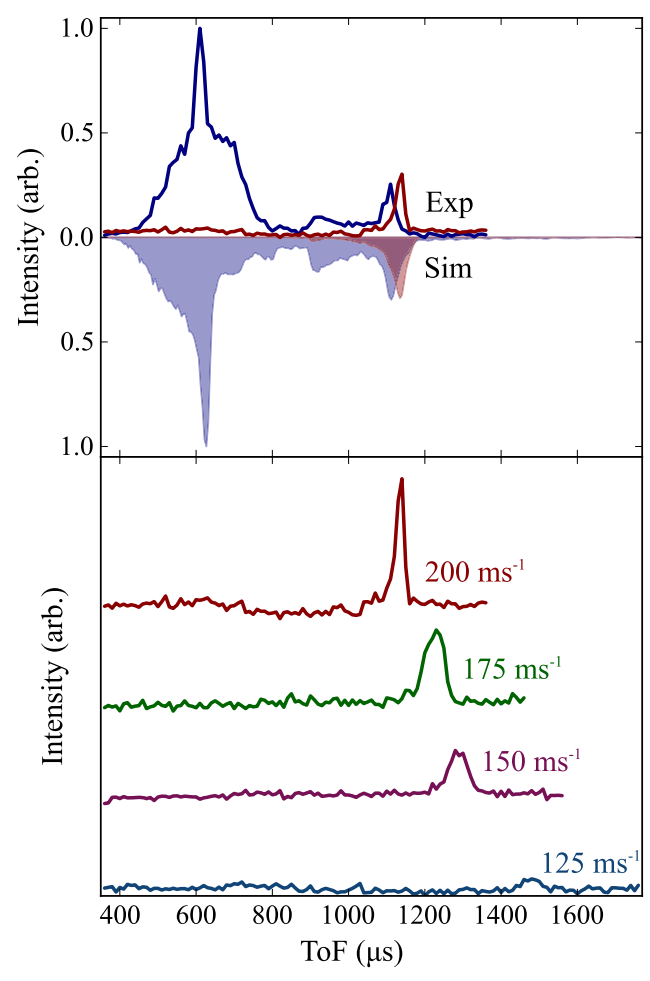

FIG. 4. Top panel: Experimental ToF distribution of $\mathrm{H}$ atoms detected at $z=319 \mathrm{~mm}$ after passing through the guide (red solid line) and when no guide is present (blue solid line), following deceleration to $200 \mathrm{~ms}^{-1}$. The corresponding simulated distributions are shown reflected below the experimental traces, with the red and blue shaded areas corresponding to the simulated ToF profiles with and without the guide present, respectively. Bottom panel: Experimental traces demonstrating that different final velocities can be targeted, spanning from $200 \mathrm{~ms}^{-1}$ down to $125 \mathrm{~ms}^{-1}$.

penetrate the beam axis can also be modified, to adjust the velocity resolution of the resulting guided beam; increasing the overlap between the blades increases the velocity resolution, at the expense of the number of particles transmitted. This trade-off can be clearly seen in Fig. 5: the trace recorded with no overlap of the blades (i.e., both blades positioned at precisely $b 1=b 2=+0.0 \mathrm{~mm}$ ) displays a higher-velocity shoulder

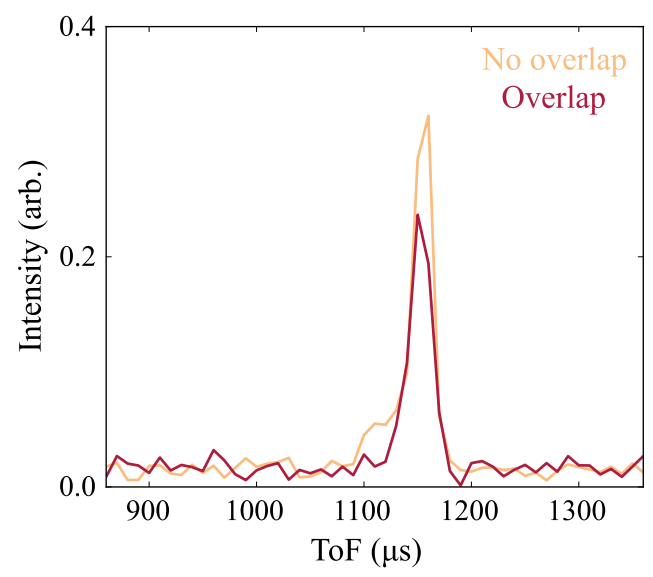

FIG. 5. Experimental ToF distribution of $\mathrm{H}$ atoms detected at $z=319 \mathrm{~mm}$ after passing through the guide: with the blades located at $b 1=b 2$ $=+0.0 \mathrm{~mm}$ (orange trace, labeled "No overlap") and with the blades in their optimised positions of $b 1=+1.4 \mathrm{~mm}, b 2=+0.0 \mathrm{~mm}$ (red trace, labeled as "Overlap"). 
alongside the decelerated peak that is not present in the trace recorded with the optimised blade overlap $(b 1=+1.4 \mathrm{~mm}$, $b 2=0.0 \mathrm{~mm})$. The lower peak intensity and narrower arrival time (due to the disappearance of the higher-velocity shoulder) in the optimised configuration are attributed to the loss of partially decelerated $\mathrm{H}$ atoms. Indeed, simulations reveal that the average velocity of the hydrogen atoms reaching the target region (at $z=350 \mathrm{~mm}$ ) when the blades are not overlapped is $275 \pm 136 \mathrm{~ms}^{-1}$, in contrast to an average velocity of 205 $\pm 9 \mathrm{~ms}^{-1}$ when the blades are overlapped in the optimised configuration.

Although the flux of $\mathrm{H}$ atoms emerging from the guide has not been experimentally determined yet, it is estimated to be comparable to the typical densities of decelerated particles obtained in conventional Zeeman decelerators, which are around $10^{8} \mathrm{~cm}^{-3} .^{29}$ Simulations indicate that about half of the particles with the target velocity emerging from the decelerator are successfully transmitted through the guide. Despite the relatively low number densities, the design of the magnetic guide is such that the user can decide upon (and modify) the optimal balance between transmission efficiency and velocity resolution for each given application in situ, based on the experimentally observed signal levels. This precise velocity filtering of radical beams is unprecedented, with the tunability in the target velocity and resolution significantly increasing the potential functionality of the device.

Furthermore, a different velocity range (beyond the current capabilities) could be targeted-or a different paramagnetic species could be addressed-by exchanging the Halbach arrays described in this work for others with different radii [internal and external, Fig. 1(a)], thickness, and magnetisation. For instance, Halbach arrays with $B_{0}=1.4 \mathrm{~T}, r_{i}=3 \mathrm{~mm}$, $r_{e}=7 \mathrm{~mm}$, and $l_{h}=7 \mathrm{~mm}$ would be suitable to target $\mathrm{H}$ atoms with final velocities between 200 and $355 \mathrm{~ms}^{-1}$, as well as $\mathrm{O}$ atoms with final velocities between 85 and $155 \mathrm{~ms}^{-1}$. The simulations used for design optimisation are readily applicable to other species provided one has the relevant Zeeman energy level diagram for that species, and in general, the existing design for $\mathrm{H}$ atoms can be used as a starting point for optimising the geometry of the setup for other species. This opens the door to many interesting potential applications that would benefit from a pure source of radicals, with a tuneable velocity and narrow velocity distribution. Indeed, most radical sources yield a mixture of radical species and precursor gas and leave little scope for control over the velocity or purity of the radicals produced.

While the magnetic guide was originally conceived to purify a Zeeman-decelerated beam of radicals, as successfully demonstrated here, it could also be employed as a stand-alone beam filter. Bent electrostatic quadrupole guides have long been employed to select those species moving with a velocity below a given threshold value from an effusive beam of dipolar particles. ${ }^{1,30}$ The magnetic guide presented here could do this - and more-by manipulating an effusive beam of radicals. Instead of transmitting all species moving below a certain threshold velocity, the flexible design of the magnetic guide will allow for narrow, and tuneable, velocity filtering of paramagnetic species from an effusive source. In this way, a diverse range of radical beams could be generated, facilitating the study of radical reactions over a wide range of collision energies.

\section{CONCLUSION}

In summary, a magnetic guide composed of four translatable Halbach arrays and two translatable skimming blades has been demonstrated to act as a beam filter such that only those radical species with a desired velocity are transmitted through the guide unhindered. Within the magnetic guide, the first two arrays are shifted in the $y$ direction compared to the beam axis by $+1.0 \mathrm{~mm}$ - to steer the $\mathrm{H}$ atoms traveling at the target velocity away from the beam axis and to collimate the deflected beam, respectively. The second pair of arrays is shifted in $y$ by $+0.6 \mathrm{~mm}$, bringing the deflected radicals back to the beam axis and collimating the radical beam once again, respectively. Two retractable blades are positioned after the second and fourth arrays to remove all particles that do not follow the trajectory of the target $\mathrm{H}$ atoms. The breaking of the $x y$ symmetry caused by the deflection away from (and back to) the beam axis allows the blades to overlap in the $y$ dimension, yielding a considerably purer $\mathrm{H}$ atom beam than can be achieved with a straight analog of the proposed guide [Fig. 2(c)]. The target velocity is tuneable and can be varied by simply translating the position of the second and fourth Halbach arrays with respect to the first and third arrays. The properties of the arrays (internal and external radii, thickness, and magnetisation) are chosen such that hydrogen atoms with velocities between $125 \mathrm{~ms}^{-1}$ and $200 \mathrm{~ms}^{-1}$ can be addressed. A different velocity range could be targeted, or a different radical species could be transmitted, by exchanging the current Halbach arrays for others with different radii, thickness, or magnetisation.

Generating a pure source of velocity-selected radical species has been an ongoing experimental challenge, inhibiting the detailed experimental study of many important processes. The flexible magnetic guide design detailed here achieves unprecedented purification of Zeeman-decelerated $\mathrm{H}$ atoms, leading to a new level of control over a beam of radicals. In this work, the Zeeman decelerator serves as a source of slow-moving $\mathrm{H}$ atoms. The versatility of the magnetic guide is such that it could also be employed as a stand-alone device (without the Zeeman decelerator); provided there are some particles in the incoming beam that exhibit the desired velocity (as in an effusive beam), the magnetic guide will transmit only those particles that fall within the selected target velocity range. As such, the magnetic guide detailed in this work has a plethora of potential ground-breaking applications. With the single-ion sensitivity that can be achieved by monitoring reactions in Coulomb crystals, combining magneticguide-filtered radicals with Coulomb-crystallized ions held within an ion trap could pioneer the precise experimental examination of many important ion-radical reactions. Further applications, in areas spanning from fundamental measurements (chemical physics) to industrially relevant reaction studies (radical-surface interactions), are envisaged to follow. 


\section{ACKNOWLEDGMENTS}

T.P.S. and B.R.H. acknowledge the financial support of the EPSRC (Project Nos. EP/N004647/1 and EP/N032950/1). J.T. acknowledges financial support from the EPSRC (Award Reference No. 1512257) and the Merton College Simms bursary. Supporting data can be obtained from the Oxford Research Archive (https://doi.org/10.5287/bodleian:ZO7qyXE69).

${ }^{1}$ S. Willitsch, M. T. Bell, A. D. Gingell, S. R. Procter, and T. P. Softley, Phys. Rev. Lett. 100, 043203 (2008).

${ }^{2}$ B. R. Heazlewood and T. P. Softley, Annu. Rev. Phys. Chem. 66, 475 (2015).

${ }^{3}$ K. A. E. Meyer, L. L. Pollum, L. S. Petralia, A. Tauschinsky, C. J. Rennick, T. P. Softley, and B. R. Heazlewood, J. Phys. Chem. A 119, 12449 (2015).

${ }^{4}$ G. Bruno, P. Capezzuto, and A. Madan, Plasma Deposition of Amorphous Silicon-Based Materials (Elsevier, 1995).

${ }^{5}$ N. G. Adams and D. Smith, Int. J. Mass Spectrom. Ion Phys. 21, 349 (1976).

${ }^{6}$ R. T. McIver, Rev. Sci. Instrum. 41, 555 (1970).

${ }^{7}$ B. R. Rowe and J. B. Marquette, Int. J. Mass Spectrom. Ion Processes 80, 239 (1987)

${ }^{8}$ N. Vanhaecke, U. Meier, M. Andrist, B. H. Meier, and F. Merkt, Phys. Rev. A 75, 031402 (2007)

${ }^{9}$ E. Narevicius, C. G. Parthey, A. Libson, J. Narevicius, I. Chavez, U. Even, and M. G. Raizen, New J. Phys. 9, 358 (2007).

${ }^{10}$ K. Hayashi, N. Sakudo, T. Noda, A. Takeda, K. Fujimura, and N. Shimizu, Nucl. Instrum. Methods Phys. Res., Sect. B 127-128, 918 (1997).

${ }^{11}$ Atomic and Molecular Beam Methods, edited by G. Scoles (Oxford University Press, 1988).

${ }^{12}$ T. Kravchuk, M. Reznikov, P. Tichonov, N. Avidor, Y. Meir, A. Bekkerman, and G. Alexandrowicz, Science 331, 319 (2011).
${ }^{13}$ Y.-P. Chang, K. Długołęcki, J. Küpper, D. Rösch, D. Wild, and S. Willitsch, Science 342, 98 (2013).

${ }^{14}$ M. Kirste, H. Haak, G. Meijer, and S. Y. T. van de Meerakker, Rev. Sci. Instrum. 84, 073113 (2013).

${ }^{15}$ J. P. Beardmore, A. J. Palmer, K. C. Kuiper, and R. T. Sang, Rev. Sci. Instrum. 80, 073105 (2009).

${ }^{16}$ B. Ghaffari, J. M. Gerton, W. I. McAlexander, K. E. Strecker, D. M. Homan, and R. G. Hulet, Phys. Rev. A 60, 3878 (1999).

${ }^{17}$ A. B. Henson, S. Gersten, Y. Shagam, J. Narevicius, and E. Narevicius, Science 338, 234 (2012).

${ }^{18}$ J. Jankunas, B. Bertsche, K. Jachymski, M. Hapka, and A. Osterwalder, J. Chem. Phys. 140, 244302 (2014).

${ }^{19}$ K. Dulitz and T. P. Softley, Eur. Phys. J. D 70, 19 (2016).

${ }^{20} \mathrm{~K}$. Halbach, Nucl. Instrum. Methods 169, 1 (1980).

${ }^{21}$ K. Halbach, Nucl. Instrum. Methods 187, 109 (1981).

${ }^{22}$ T. Cremers, S. Chefdeville, N. Janssen, E. Sweers, S. Koot, P. Claus, and S. Y. T. van der Meerakker, Phys. Rev. A 95, 043415 (2007).

${ }^{23}$ A. P. P. van der Poel, K. Dulitz, T. P. Softley, and H. L. Bethlem, New J. Phys. 17, 055012 (2015).

${ }^{24}$ K. Dulitz, M. Motsch, N. Vanhaecke, and T. P. Softley, J. Chem. Phys. 140, 104201 (2014).

${ }^{25}$ J. Toscano, A. Tauschinsky, K. Dulitz, C. J. Rennick, B. R. Heazlewood, and T. P. Softley, New J. Phys. 19, 083016 (2017).

${ }^{26}$ J. Chavanne, Radia (version 4.29), http://www.esrf.eu/Accelerators/ Groups/InsertionDevices/Software/Radia (2009).

${ }^{27}$ P. Elleaume, O. Chubar, and J. Chavanne, in Proceedings of the 1997 Particle Accelerator Conference (PAC-97) (IEEE, 1997), Vol. 3, p. 3509.

${ }^{28}$ O. Chubar, P. Elleaume, and J. Chavanne, J. Synchrotron Radiat. 5, 481 (1998).

${ }^{29}$ E. Narevicius and M. G. Raizen, Chem. Rev. 112, 4879 (2012).

${ }^{30}$ S. A. Rangwala, T. Junglen, T. Rieger, P. W. H. Pinkse, and G. Rempe, Phys. Rev. A 67, 043406 (2003). 